\title{
Emergency Department Capabilities in the Kingdom of Swaziland Africa
}

\author{
Article by Erika Phindile Chowa ${ }^{1}$, Patrick Masitsela Mhlanga ${ }^{2}$, Janice A. Espinola ${ }^{3}$, Ashley F. \\ Sullivan $^{4}$, Carlos A. Camargo ${ }^{5}$ \\ ${ }^{1}$ Department of Emergency Medicine, Emory University, Atlanta, GA, USA \\ 2, 4, 5 Emergency Preparedness \& Disaster Risk Management for Health, Ministry of Health \\ Mbabane, Swaziland \\ ${ }^{3}$ Department of Emergency Medicine, Massachusetts General Hospital, Boston, MA, USA \\ E-mail: erikachowa@gmail.com ${ }^{1}$,masitselamhlanga@gmail.com ${ }^{2}$
}

\begin{abstract}
Introduction: Emergency care is available in many forms in Swaziland and to our knowledge, there has never been a systematic study of emergency departments (EDs) in the country. The purpose of this study was to describe the characteristics, resources and capacity of emergency departments (EDs) in the Kingdom.

Methods: The National Emergency Department Inventory (NEDI)-International survey instrument (www.emnet-nedi.org) was used to survey all Swaziland EDs accessible to the general public 24/7. ED staff were asked about calendar year 2014. Data were entered directly into Lime Survey, a free, webbased, open-source survey application. Responses were analyzed using descriptive statistics, including proportions and medians with interquartile ranges (IQR).

Results: Sixteen of 17 EDs participated (94\% response rate). Participating EDs were in either in hospitals (69\%). EDs had a median of 53,399 visits per year (IQR 15,000 to 97,895). Fourteen (88\%) EDs had a contiguous layout and the other $2(12 \%)$ were noncontiguous. Overall, $8(53 \%)$ had access to cardiac monitors and 11 (69\%) had a 24/7 clinical laboratory available. Only 1 (6\%) ED had a dedicated CT scanner, while 2 (13\%) others had limited access through their hospital. The typical ED length-of-stay was between 1 and 6 hours (44\%). The most commonly available specialists were general surgeons, with 9 (56\%) EDs having them available for in-person consultation. No EDs had a plastic surgeon or psychiatrist available. Overall, $75 \%$ of EDs reported running at overcapacity.

Conclusions: Swaziland EDs were predominantly contiguous and running at overcapacity, with high patient volumes and limited resources. The limited access to technology and specialists are major challenges. We believe that these data support greater resource allocation by the Swaziland government to the emergency care sector.
\end{abstract}

\section{Introduction}

The Kingdom of Swaziland is a landlocked country in southern Africa with a surface area of approximately $17,000 \mathrm{~km}^{2}$. The population is approximately 1.3 million people; $53 \%$ of the population being female. ${ }^{2}$ According to the World Bank, Swaziland, with its gross domestic product of $\$ 3,350$ per capita in 2014, is in the lower middle-income category of countries for this indicator. ${ }^{2}$ The health status in Swaziland is below expectations, with life expectancy at birth estimated at only 53 years. ${ }^{3}$ The number one cause of death in the country is HIV (27\% prevalence, which is highest in the world) followed by lower respiratory infections, tuberculosis, stroke, and diarrheal illness. ${ }^{3,4}$

With this heavy burden of disease, the Swaziland government has created public health strategies to improve mortality rates within the country. For example, as a response to having one of the highest percentages of road traffic accidents in the world, the Ministry of Health established the Emergency Preparedness and Risk Management Department (EPR) in 2008 to create a public pre-hospital care service to respond to emergencies and facilitate inter-department transfers. ${ }^{5}$ It remains unclear what 
DOI: $10.21522 / \mathrm{TIJPH} .2013 .05 .04 . A r t 048$

ISSN: $2520-3134$

impact this has had on the mortality rates, but the EPR has brought attention to emergency care within the country. Although emergency medicine as a specialty is still non-existent, emergency care is available in many forms. Until the current study, there has never been, to our knowledge, a systematic study of emergency departments (EDs) in Swaziland.

The objective of this study was to describe the characteristics, resources and capacity of EDs in the Kingdom of Swaziland. Such information would provide a valuable benchmark for future efforts to improve the accessibility and quality of emergency care.

\section{Methods}

Ethical approval in Swaziland was obtained from the Swaziland Scientific and Ethics Committee of the Ministry of Health. The Partners Healthcare Institutional Review Board (IRB) also reviewed study and determined it to be exempt.

This cross-sectional study utilized a series of surveys developed by the Emergency Medicine Network (EMNet) in Boston, Massachusetts (www.emnet-nedi.org) to assess emergency department (ED) characteristics and capabilities in the international setting. ${ }^{6}$ For the purpose of this study, we defined an ED as any emergency care facility that was open 24 hours/day, 7 days/week and provided evaluation and management of critically ill and injured patients at the earliest stages of medical crisis. This included casualty units, medical and surgery units that received emergencies throughout the day. Specialty facilities (e.g. Swaziland's Psychiatric and TB hospital) were excluded given care was only provided to a specific population. A list of all emergency facilities in the country was provided by the Ministry of Health. Seventeen EDs were determined to meet this inclusion criteria. The matron (Senior Nursing Officer) of each ED or senior clinical officer (Medical Doctor) were identified from each ED to participate in survey. Research staff met with these individuals and administered the survey in-person. All questions were asked in reference to the calendar year 2014.

Data was entered directly into Lime Survey (https://www.limesurvey.com); a free, web-based, opensource survey application for online data collection. Responses were exported into an Excel spreadsheet (Microsoft Corp, Redmond, WA) and descriptive analyses were performed using Stata 14.1 (Stata Corp, College Station, TX). Data are presented as proportions and medians with interquartile ranges (IQR).

\section{Results}

Sixteen of 17 Swaziland EDs completed the survey (94\% response rate). Despite multiple phone calls and in-person visits, the one missing ED did not complete the survey. The participating EDs were located in either hospitals $(69 \%)$ or health centers $(31 \%)$. Fourteen (88\%) EDs were contiguous (i.e., one unified area to see emergencies) and $2(12 \%)$ were noncontiguous (Figure 1). Among contiguous EDs, 11 (79\%) treated emergencies in the hospital's outpatient department (OPD) and $3(21 \%)$ in other dedicated emergency units. Overall, EDs had a median volume of 53,399 visits per year (IQ 15,000 to 97,895), with the highest patient volume being 206,265 per year. No hospital saw adults only or children only. A physician was available in-person 24/7 in $88 \%$ of EDs and a nurse in $94 \%$ of EDs. The median number of hospital beds was 51 (IQR 35-210) and ED beds 4 (IQR 3-12). Overall, 75\% of EDs perceived being overcapacity.

ED length-of-stay varied; 38\% of EDs had an average length-of-stay of less than 1 hour, 44\% with 1-6 hours, and $19 \%$ with $>6$ hours. Most (69\%) EDs had $<20 \%$ of visits come in by ambulance and most $(63 \%)$ reported that $\geq 80 \%$ of hospital admissions came through the ED.

Available resources were limited (Figure 2). Three hospitals reported having CT scanners, but only one hospital reported a dedicated scanner for the ED. No hospital had a respiratory isolation room. In-person consultants were available in Swaziland EDs, and included surgeons, obstetricians, anesthesiologist, cardiologists, neurosurgeons, neurologist and orthopedic surgeons. By contrast, plastic surgeons and psychiatrists were not available in any ED and general surgeons were available in only 56\% of EDs. Most specialists were not available 24/7 (Figure 3). 


\section{Discussion}

The Kingdom of Swaziland handles health emergencies in diverse healthcare settings. Although next to South Africa, where emergency medicine became a specialty in 2003, Swaziland still does not recognize emergency medicine as a specialty and emergency care is not standardized across hospitals. While $88 \%$ of EDs in Swaziland were contiguous, most (79\%) overlapped with the hospital's OPDs. The noncontiguous EDs saw emergencies in a combination of areas in the hospital (e.g. OPDs, casualty units, surgical units, maternity and pediatrics). We were surprised to see that emergencies within the country were mostly seen in hospital OPDs which also see non-emergent cases and provide public health interventions (e.g. immunizations and family planning). For example, this differs from the neighboring country of South Africa were emergencies are seen in emergency centers that do not see primary care visits. $^{7}$ As a result, emergencies in Swaziland OPDs are not distinguished from primary care visits in annual reports. The median volume of 53,399 visits per year might be smaller for actual emergency visits, as commonly seen in the US or Europe. Some EDs reported high patient volumes (e.g. 200,000+ in one ED) and that cannot be entirely attributed to OPD primary care visits. For example, one ED with patient volumes in excess of 200,000 is a referral hospital and receives emergencies from distant clinics and health centers. Other reasons for high ED volumes could be the result of poor utilization of more traditional primary care clinics and lack of public education on when to present to the ED, but reasons for this were not formally evaluated and require further study. However, if poor utilization is the cause, investing time and resources into primary care clinics and educating the public could be critical in offloading crowded EDs. Hospital resources could then be reserved for true emergencies and hospitalized patients.

Emergencies were not only seen in hospitals, but in health centers as well. Hospitals are in the various regions of the country, while health centers are mostly in rural areas within 2 hours of the hospitals. Hospital EDs are more equipped than health centers, but also share similar problems. Almost all hospitals have a surgeon on call who can deal with a wide range of surgical emergencies. More specialized surgeons, such as neurosurgeons and orthopedists, are located in the major hospitals but still rare - e.g., $18 \%$ of EDs have neurosurgeons available 24/7 and 19\% of EDs have orthopedists available 24/7. With trauma secondary to road traffic accidents among top causes of death in the country, these consultants are crucial and their limited availability is of major concern. Plastic surgeons and psychiatrist are unavailable across all Swaziland EDs; the conditions these specialists treat may not seem as crucial as neurosurgeons, general surgeons, obstetricians or orthopedic surgeons, but their absence from EDs may contribute to long-term disability and morbidity for many patients. This shortage of specialist availability is certainly not unique to Swaziland. Currently Swaziland deals with the lack of specialists by providing programs to refer stable patients to South Africa who require specialized care. However, this is not an option when faced with an unstable patient. Consequently, our study indicates the need to improve specialist availability for Swaziland EDs.

Health centers provide primary care needs for the public, but like hospitals they are open 24/7 for emergencies and have inpatient beds. They are necessary for the care of many individuals who have emergencies and may not be able to get to hospitals in a timely fashion because of transportation. Unfortunately, many of these health centers lack technological resources or specialist to treat most emergencies. Our national study revealed that none of the health centers had cardiac monitors or mechanical ventilators available for their departments. Critically ill trauma patients were being treated without these resources. Consultants were scarce, with none of the health centers having access to neurosurgeons, orthopedists or plastic surgeons available for consultation. Complex patients are routinely transferred to the nearest referral hospital for definitive management. The observed lack of resources at most of these health centers seems likely to have an adverse impact on patient care. Indeed, some of these health centers are quite far from referral hospitals and waiting for transport (without proper equipment or resources to stabilize sick patients) is likely to have led to serious morbidity or mortality. 
DOI: $10.21522 / \mathrm{TIJPH} .2013 .05 .04 . A r t 048$

ISSN: $2520-3134$

All EDs lacked adequate technological resources. Overall, 53\% of EDs had available cardiac monitors and $19 \%$ had mechanical ventilators; $69 \%$ had a 24/7 laboratory with capability of performing a stat blood gas with potassium. Health care funds to provide for these essential ED items need to be a priority, especially in distant health centers where clinicians have no choice but to keep patients overnight for transfer in the morning. With only three CT scans in the country, we were not surprised to learn that only one ED had a dedicated CT scanner. All other EDs shared their CT with the inpatients. Hospitals should consider prioritizing emergency patients in radiology queues and ensuring that EDs are within proximity to the available CT scan within that hospital. Lastly, and perhaps of greatest concern, is the lack of a negative pressure room in all EDs. As listed above, tuberculosis is among the top 5 causes of death in the country and, even though Swaziland has a TB-dedicated hospital, this does not protect EDs from seeing patients with undiagnosed TB. With the repercussions of this disease and other air borne illnesses, the lack of a negative pressure room in EDs could pose a major public health threat in a country of more than 1 million people. This shortcoming requires urgent attention from government since negative pressure room should be a requirement for all Swaziland EDs.

With the longstanding limited resources to care for emergency patients, it's possible that nurses and physicians are comfortable with caring for sick patients in this austere environment. Although there are currently no public medical schools or formal emergency training programs for physicians in the country, training for all providers who work in EDs can come in the form of formalized emergency education such as special courses and "in-service" training. This does not solve the shortage of available specialists, but with medical training in emergency care, providers can learn to more effectively stabilize patients and prepare them for transfer to tertiary hospitals where the few specialist are available. We did not evaluate emergency medical care training amongst nurses and physicians, but plan to pursue such work in the years ahead.

\section{Conclusions}

Swaziland EDs are primarily contiguous with high visit volumes and few ED beds. Many EDs reported being overcapacity and lacking technological resources. We believe that these challenges in emergency care could be addressed with improved resource allocation to the emergency care sector in the country. Other options include importing specialists to the country and promoting emergency care training among all ED staff.

\section{Acknowledgments}

This project was made possible by the staff at the Ministry of Health Swaziland and the Department of Emergency Preparedness and Disaster Risk Management for Health in Swaziland. We thank the ED directors in Swaziland for their participation in the study.

\section{References}

[1].CIA Factbook. Swaziland 2014. Available at: https://www.cia.gov/library/publications/the-worldfactbook/geos/wz.html (Last accessed 7/15/20106).

[2].Emergency Preparedness Response. Division of the Ministry of Health. Available at: www.epr.org.sz (Last accessed 2/27/2016).

[3].Steptoe AB, Corel B, Sullivan AF, et al. Characterizing emergency departments to improve understanding of emergency care systems. Int J Emerg Med 2011; 4:42e50.

[4].The Emergency Medicine Network. The National ED Inventories Survey Instrument. Available at: http://www.emnet-nedi.org (Last Accessed 10/1/2016).

[5].Wallis L, Garach S, Kropman A. State of emergency medicine in South Africa. Int J Emerg Med 2008; 1(2) p6971.

[6].World Bank. Swaziland Country Profile. 2014. Available at: http://Data.worldbank.org/country/swaziland (Last accessed 2/27/2016). 
[7].World Health Organization. Swaziland: WHO Statistical Profile 2012. Available at: www.who.int/gho/countries/swz (Last accessed 2/27/2016).

\section{Tables and figures}

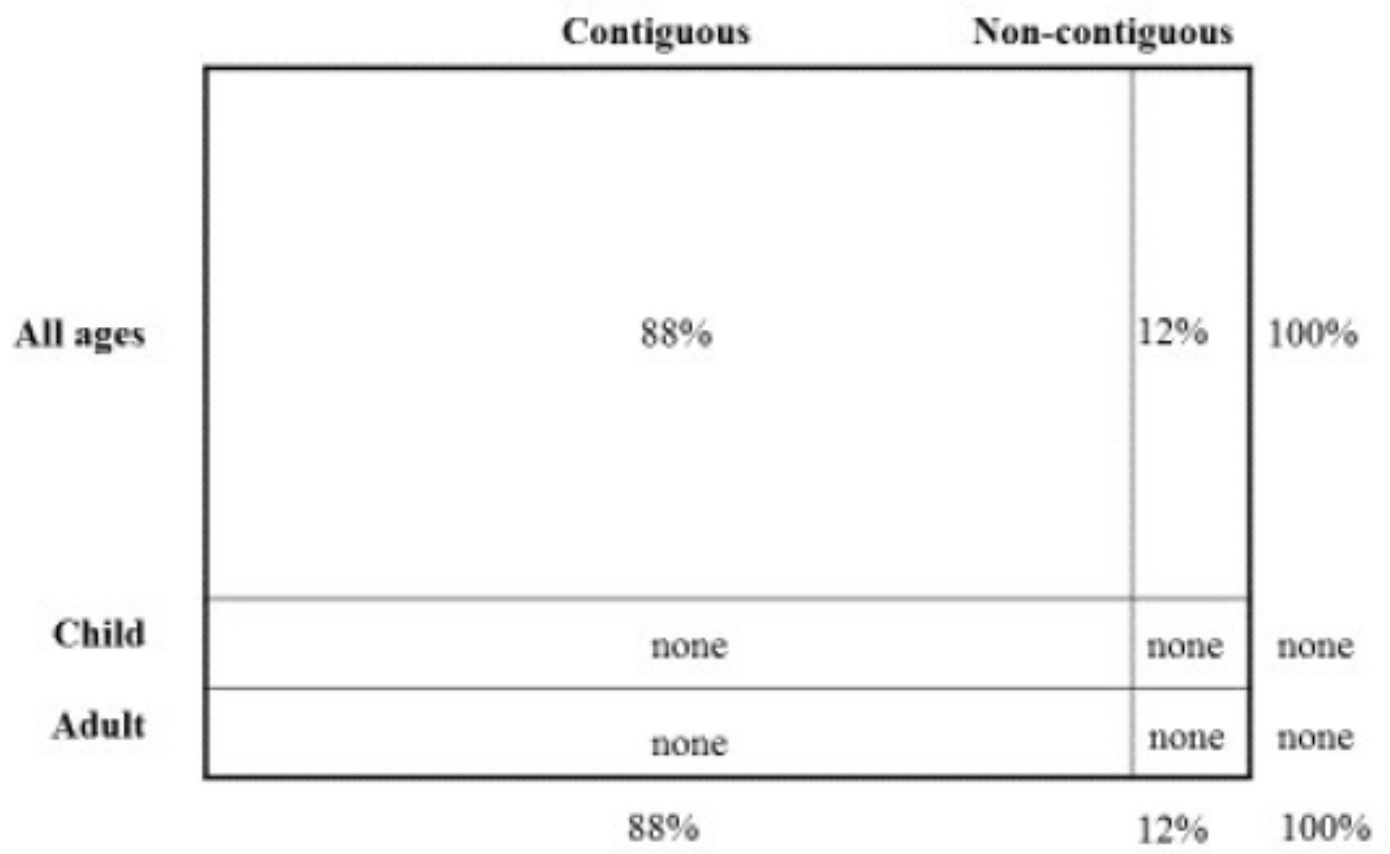

Figure 1. Snapshot of overall emergency department (ed) characteristics in swaziland

Figure Legend:

$16 / 17$ EDs (94\% response)

$910 \mathrm{ED}$ visits per 1,000 population 
DOI: $10.21522 / \mathrm{TIJPH} .2013 .05 .04 . A r t 048$

ISSN: $2520-3134$

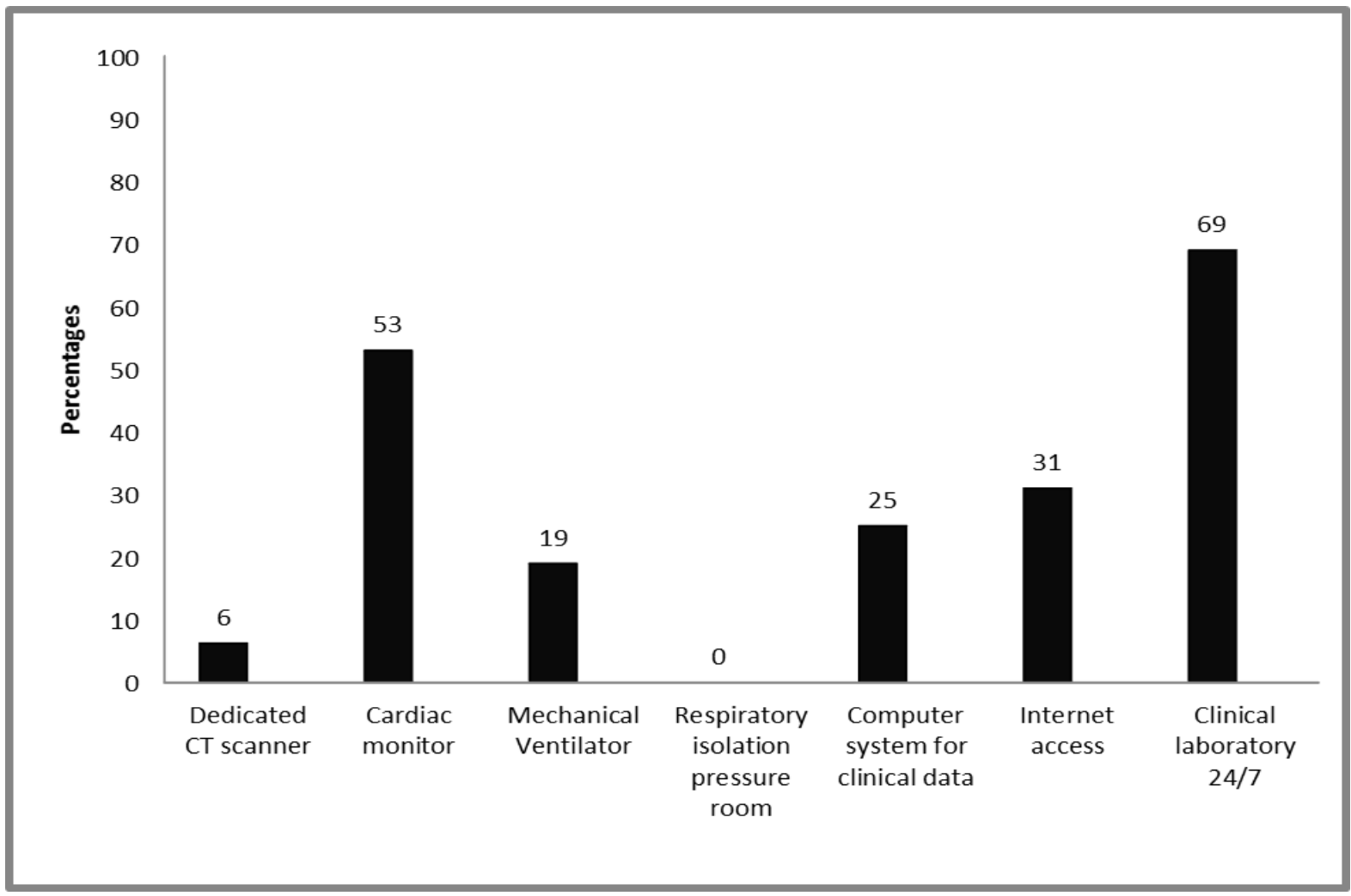

Figure 2. Percentage of emergency departments with available resources

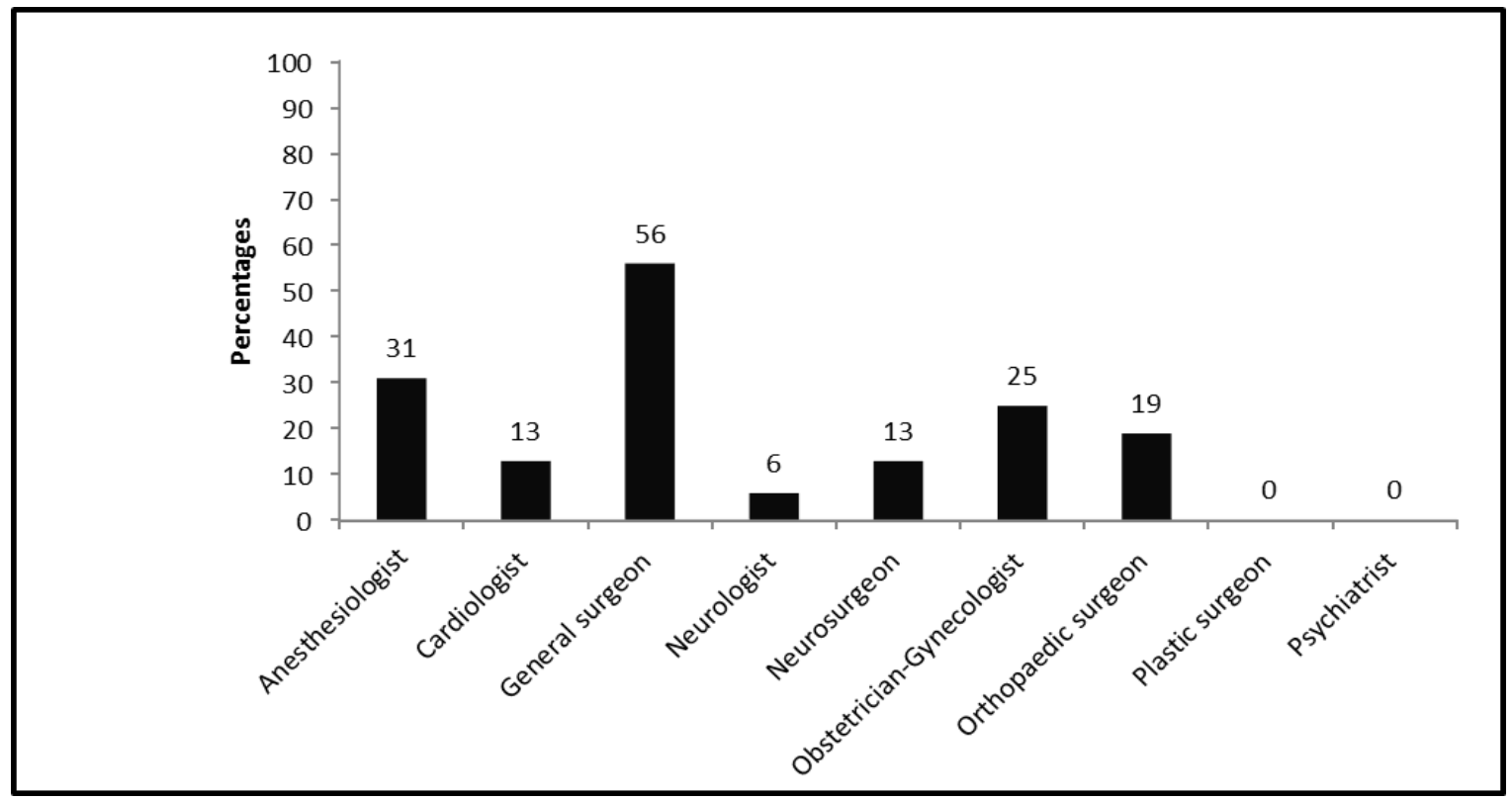

Figure 3. Percentage of emergency departments with specialists available 24/7 